\title{
REVIEW
}

\section{Analgesia in the emergency department: a GRADE-based evaluation of research evidence and recommendations for practice}

\author{
Chris Lipp*, Raj Dhaliwal, Eddy Lang \\ This article is one of ten reviews selected from the Annual Update in Intensive Care and Emergency Medicine 2013 and co-published as a series \\ in Critical Care. Other articles in the series can be found online at http://ccforum.com/series/annualupdate2013. Further information about the \\ Annual Update in Intensive Care and Emergency Medicine is available from http://www.springer.com/series/8901.
}

\section{Introduction}

Emergency physicians care for patients with pain on an extremely frequent basis [1-20]. The prevalence of pain as the presenting complaint of patients seeking emergency department (ED) care ranges from $38 \%$ [3] to as high as $78 \%$ [1]. As a result, evidence-based use of analgesics should be a foundational skill of emergency physicians. However, the literature consistently reports that emergency physicians are often poor at treating pain $[1,2,8,13,14,16,19,20]$. Notwithstanding the prevalence of pain in the ED, many patients often report that their pain was not properly treated [3,7,20]. In addition to a compromised patient experience, sub-optimal treatment of pain will result in decreased department flow, increased wait times, more return visits to the ED, and increased hospitalization rates.

Very few evidence-based resources and guidelines exist to inform emergency physicians on how to treat pain. One recent guideline on acute pain management compiled by the college of Anaesthetists of Australia and New Zealand [21] was focused primarily on treating pain perioperatively and did not include stratified or graded recommendations based on the literature, highlighting the paucity of emergency medicine-specific guidance. Emergency physicians need an effective, evidenced-based approach to analyze and apply the options available for acute pain management.

The objective of this article is to synthesize and evaluate the quality of medical literature surrounding analgesia delivery in the adult ED using the Grading Assessment, Development and Evaluation (GRADE) framework. We further strived to provide emergency physicians with

*Corresponding author: ctlipp@ucalgary.ca

University of Calgary, Faculty of Medicine, Alberta Health Services, Calgary, Canada graded recommendations upon which analgesics should be used to treat adults with acute pain in the ED.

\section{Question formulation}

Prior to searching the literature we developed seven clinically-oriented questions based on a scoping of the literature and a review of locally utilized ED analgesic order sets. This initial surveying of medical literature and our local practice environment allowed us to identify the most commonly prescribed intravenous and oral analgesics used in adult EDs in our health care region.

We then used the patient-intervention-comparisonoutcome (PICO) approach to develop our seven research questions (Table 1). This approach has been adopted by many authors of systematic reviews and guideline panels, including the International Liaison Committee on Resuscitation (ILCOR) and the American College of Chest Physicians (ACCP) [22-24]. It involves identifying a specific population or setting to which recommendations may be applied. Subsequently each question compares two specific management strategies (intervention and comparison). Finally, we defined important patient oriented outcomes (e. g., change in pain) as well as any adverse effects of the medication or other safety concerns.

All seven draft questions were reviewed and revised through an iterative process involving all authors. These seven clinically-based PICO questions compared analgesics (morphine, fentanyl, hydromorphone, nonsteroidal anti-inflammatory drugs [NSAIDs], codeine, oxycodone) commonly used in the management of adult acute pain in the ED. The primary critical outcome across all PICO questions was a clinically significant change in pain using validated instruments, such as a visual analog (VAS) or numeric rating scale (NRS). The time frame used to assess change in pain varied from 30 minutes to 
Table 1. The seven questions developed for analysis

\begin{tabular}{|c|c|c|}
\hline \multicolumn{2}{|r|}{ Question (PICO format: Population-Intervention-Comparison-Outcome) } & \multirow{2}{*}{$\begin{array}{l}\text { Route } \\
\text { Intravenous }\end{array}$} \\
\hline 1. & $\begin{array}{l}\text { For adults accessing the emergency department with acute pain, should parenteral morphine or fentanyl be used to manage } \\
\text { acute moderate-severe pain based on reported change in pain using the visual analog scale? }\end{array}$ & \\
\hline 2. & $\begin{array}{l}\text { For adults accessing the emergency department with acute pain, should parenteral hydromorphone or morphine be used to } \\
\text { manage acute severe pain based on reported change in pain using the visual analog scale? }\end{array}$ & Intravenous \\
\hline 3. & $\begin{array}{l}\text { For adults accessing the emergency department with acute pain, should a parenteral hydromorphone } 1+1 \mathrm{mg} \text { patient-driven } \\
\text { protocol or other intravenous opioids at any dose (physician-driven protocol) be used to manage acute pain based on reported } \\
\text { change in pain using the visual analog scale? }\end{array}$ & Intravenous \\
\hline 4. & $\begin{array}{l}\text { For adults accessing the emergency department with acute pain who do not need parenteral analgesia but request an } \\
\text { analgesic for outpatient pain management, should oral hydromorphone or oxycodone be used to treat acute pain based on } \\
\text { patient reported efficacy (change in pain) and adverse side effects? }\end{array}$ & Oral \\
\hline 5. & $\begin{array}{l}\text { For adults accessing the emergency department with acute pain who do not need parenteral analgesia but request an } \\
\text { analgesic for outpatient pain management, should non-specific NSAIDs (e. g., ibuprofen) or codeine-acetaminophen be used } \\
\text { for mild-moderate acute pain based on patient reported efficacy (change in pain) and adverse side effects? }\end{array}$ & Oral \\
\hline 6. & $\begin{array}{l}\text { For adults accessing the emergency department with acute pain who do not need parenteral analgesia but request an } \\
\text { analgesic for outpatient pain management, should COX-2 specific NSAIDs (e. g., celecoxib) or codeine-acetaminophen be used } \\
\text { for mild-moderate acute pain based on patient reported efficacy (change in pain) and adverse side effects? }\end{array}$ & Oral \\
\hline 7. & $\begin{array}{l}\text { For adults accessing the emergency department with acute pain who do not need parenteral analgesia but request an } \\
\text { analgesic for outpatient pain management, should oxycodone-acetaminophen or codeine-acetaminophen be given to } \\
\text { patients with acute pain based on patient reported efficacy (change in pain) and adverse side effects? }\end{array}$ & Oral \\
\hline
\end{tabular}

NSAID: non-steroidal anti-inflammatory drug; COX: cyclooxygenase

two hours, depending on the medication route of administration. Secondary patient-oriented outcomes included serious adverse events, patient satisfaction, and side effects. Serious adverse events were defined as respiratory depression (less than 12 breaths per minute), decline in pulse oximetry oxygen saturation to less than $92 \%$, decline in systolic blood pressure below $90 \mathrm{mmHg}$, or the need for administration of naloxone after opioid administration.

\section{Search strategy}

After developing the seven PICO questions we conducted searches for each question using PubMed, Ovid MEDLINE, EMBASE, the Cochrane Database of Systematic Reviews, and the TRIPdatabase. These databases were systematically searched following consultation with a professional health sciences librarian who assisted us in our search methodology and use of specific keywords including Medical Subject Headings (MeSH) terms (Table 2). Our search took place over a six-month period of time, from January to June 2011. We conducted seven unique searches using the same search keywords as well as the various names of the intervention and comparison drugs in each question. When possible, we used MeSH terms for searching the U.S. National Library of Medicine. Prior to searching the databases we also developed inclusion and exclusion criteria (Table 2) to assist us in focusing each literature search.

The results of each literature search were saved and a single reviewer screened titles, flagging articles that met the inclusion criteria based on the search keywords and terms.

Articles flagged as relevant in the initial screen were selected for abstract review. Abstracts for each PICO question were screened by the same reviewer and when abstracts met the inclusion criteria, a full text copy of the article was retrieved.

\section{Use of GRADE}

In our analysis of the situation we decided to use the GRADE framework, which is becoming the benchmark for communicating evidence-based medicine throughout the world [25]. Numerous prominent health organizations, including the World Health Organization (WHO), the ACCP, the Society of Critical Care Medicine (SCCM), the National Institute for Clinical Excellence (NICE, UK), and the European Respiratory Society (ERS) have endorsed or adopted the GRADE approach as a means of analyzing medical evidence and developing guidelines.

We used the GRADE system and its quality of evidence criteria as well as GRADE-Pro software to analyze the articles for each PICO question. This software-supported approach is used in the GRADE methodology and enables clinician-researchers to succinctly analyze structured clinical questions using the GRADE methodology [26,27]. GRADE classifies evidence into four categories (high, moderate, low and very low) based on a body of evidence related to a specific outcome. This classification is based on an assessment of the research studies in question, including assessing for bias, indirectness, imprecision, 
Table 2. Search keywords, inclusion and exclusion criteria for literature searches

\begin{tabular}{lll}
\hline Search Terms & Inclusion Criteria & Exclusion Criteria \\
\hline Pain* & Randomized controlled trials & Case studies, primary research \\
Therapy & Systematic reviews & Published prior to the year 1990 \\
Treatment & Meta-analyses & Oncology-related treatments \\
Emergency medicine* & Clinical trials & \\
Emergency service hospital* & Cohort studies & \\
Adult* & Published from year 1990-present & \\
Analgesia & Adults (18 years and older) & \\
Therapeutics* & &
\end{tabular}

* MeSH search terms

inconsistency, and publication bias. Subsequently, the software assists the researcher through a multifactorial process of compiling the results of each study for the outcomes studied in the research question. The software then summarizes all the pertinent details of the research question and each outcome in a summary of findings
(SoF) table. Each SoF table outlines the level of evidence for each question (high, moderate, low, or very low) and serves as the basis for making recommendations on each clinical question.

Following the grading of the evidence for each of the seven PICO questions, seven separate SoF tables were

Table 3. The summary of findings table for PICO \#2: Hydromorphone (i.v.) vs. morphine (i.v.) for acute severe pain in the emergency department

\begin{tabular}{|c|c|c|c|c|c|}
\hline \multirow[b]{2}{*}{ Outcomes } & \multirow{2}{*}{$\begin{array}{l}\text { No of } \\
\text { patients } \\
\text { (studies) } \\
\text { Follow-up }\end{array}$} & \multirow{2}{*}{$\begin{array}{l}\text { Quality of } \\
\text { the evidence } \\
\text { (GRADE) }\end{array}$} & \multirow{2}{*}{$\begin{array}{l}\text { Relative } \\
\text { effect } \\
(95 \% \mathrm{Cl})\end{array}$} & \multicolumn{2}{|c|}{$\begin{array}{l}\text { Anticipated absolute effects } \\
\text { Time frame is } 30 \mathrm{~min}\end{array}$} \\
\hline & & & & Risk with morphine & $\begin{array}{l}\text { Risk difference with } \\
\text { hydromorphone }(95 \% \mathrm{Cl})\end{array}$ \\
\hline $\begin{array}{l}\text { Change in pain score } \\
\text { Mean change in numeric rating scale (NRS) } \\
{[0=\text { no pain; } 10=\text { worst pain possible }]} \\
\text { scale from } 0-10\end{array}$ & $\begin{array}{l}374 \\
\text { (2 studies) } \\
2 \mathrm{~h}\end{array}$ & $\begin{array}{l}\oplus \oplus \oplus \mathrm{O} \\
\text { MODERATE }{ }^{2,3} \\
\text { due to indirectness }\end{array}$ & & $\begin{array}{l}\text { The mean change in pain } \\
\text { score ranged across control } \\
\text { groups from 3.3-4.1 NRS }\end{array}$ & $\begin{array}{l}\text { The mean change in pain } \\
\text { score in the intervention } \\
\text { groups was } 0.9 \text { higher }(0.35 \\
\text { to } 1.75{\text { higher })^{1}}^{1}\end{array}$ \\
\hline $\begin{array}{l}\text { Serious adverse events } \\
\text { respiratory depression }(\mathrm{RR}<12) \text { or } \\
\mathrm{SpO} 2<95 \% \text { or systolic blood pressure } \\
(\mathrm{SBP})<90 \mathrm{mmHg} \text { or administration of } \\
\text { naloxone after opioid administration }\end{array}$ & $\begin{array}{l}374 \\
\text { (2 studies) } \\
2 \mathrm{~h}\end{array}$ & $\begin{array}{l}\oplus \oplus 0 \bigcirc \\
\mathrm{LOW}^{3,4} \\
\text { due to indirectness, } \\
\text { imprecision }\end{array}$ & $\begin{array}{l}\text { RR } 0.96 \\
(0.91-1.02)\end{array}$ & 54 per 1,000 & $\begin{array}{l}2 \text { fewer per 1,000 } \\
\text { (from } 5 \text { fewer to } 1 \text { more) }^{4}\end{array}$ \\
\hline $\begin{array}{l}\text { Adverse effects } \\
\text { Nausea or vomiting or pruritus }\end{array}$ & $\begin{array}{l}374 \\
(2 \text { studies }) \\
2 \mathrm{~h}\end{array}$ & $\begin{array}{l}\oplus \oplus O O \\
L O W^{3,4} \\
\text { due to indirectness, } \\
\text { imprecision }\end{array}$ & $\begin{array}{l}\text { RR } 0.96 \\
(0.83-1.1)\end{array}$ & 299 per 1,000 & $\begin{array}{l}12 \text { fewer per 1,000 } \\
\text { (from } 51 \text { fewer to } 30 \text { more) }\end{array}$ \\
\hline
\end{tabular}

The basis for the assumed risk (e. g., the median control group risk across studies) is provided in footnotes. The corresponding risk (and the $95 \%$ confidence interval $[\mathrm{Cl}]$ ) is based on the assumed risk in the comparison group and the relative effect of the intervention. RR: risk ratio

GRADE Working Groups grades of evidence

High quality: $\quad$ Further research is very unlikely to change our confidence in the estimate of effect

Moderate quality: $\quad$ Further research is likely to have an important impact on our confidence in the estimate of effect and may change the estimate

Low quality: $\quad$ Further research is very likely to have an important impact on our confidence in the estimate of effect and is likely to change the estimate

Very low quality: We are very uncertain about the estimate

${ }^{1}$ At 30 min, one study had a between-group NRS difference of $1.3(0.5-2.2)$ in favor of hydromorphone.

${ }^{2}$ One of the studies did not do a Mini Mental State Examination (MMSE) on the elderly patients; and the other cited that the hydromorphone group had a higher baseline pain score.

${ }^{3}$ Both studies were conducted in an underserviced inner city hospital with $45-60 \%$ of the patients of Hispanic descent.

${ }^{4}$ the confidence interval from the calculated relative risk is quite wide. 


\section{Intravenous analgesics}

\section{Should morphine or fentanyl be used for acute moderate-severe pain?}

We recommend fentanyl ( $1 \mathrm{mcg} / \mathrm{kg}$, then $30 \mathrm{mcg}$ q $5 \mathrm{~min}$ ) over morphine (weak recommendation, low quality evidence)

If morphine is used to treat acute pain, we suggest giving $0.1 \mathrm{mg} / \mathrm{kg}$, then $0.05 \mathrm{mg} / \mathrm{kg}$ at $30 \mathrm{~min}$, with the maximum suggested dose of $10 \mathrm{mg}$

\section{Rationale [28-30]}

- People with morphine allergies do not have allergies to fentanyl

- Fentanyl has a shorter onset of action as well as being 100 times more potent than morphine, and thus is better suited to treat acute moderate to severe pain. (Fentanyl is more lipid soluble and thus has higher bioavailability)

- There is no substantial cost difference between the two medications

- Fentanyl is reported to be less pro-emetic than morphine and does not produce a histamine release like morphine does. This leads to less hypotension and less pruritus, facial flushing, or urticaria

- Fentanyl with its 2-3 min onset and 30-60 min duration is less likely to cause prolonged sedation, and may encourage more frequent reassessment of ill patients

- Fentanyl has less of a dose stacking risk than morphine. This is especially relevant in patients with renal failure in whom morphine's metabolite accumulates, whereas fentanyl does not.

Currently the order sets have general doses of morphine at $2.5 \mathrm{mg}$ or $5 \mathrm{mg}$, and fentanyl at $50 \mathrm{mcg}$. Because adults vary in weight, ED physicians may be well served to estimate the patient's weight and dose based on that For example a $70 \mathrm{~kg}$ patient should be given $7 \mathrm{mg}$ of morphine or $70 \mathrm{mcg}$ of fentanyl as an initial loading dose (assuming there is no contraindication to a high loading dose)

2. Should hydromorphone or morphine be used for acute severe pain in the emergency department?

We recommend hydromorphone $(0.015 \mathrm{mg} / \mathrm{kg}$ i.v.) as a comparable, potentially superior, analgesic to morphine $(0.1 \mathrm{mg} / \mathrm{kg}$ i.v.) (strong recommendation, moderate quality evidence)

Rationale $[28,31,32]$

- Hydromorphone has a quicker onset of action, when compared with morphine

- Hydromorphone is comparable in cost to morphine

- Morphine, with a longer onset of action and greater risk for dose stacking, places patients at a higher risk for toxicity (in the context of renal failure) and hypoventilation or, on the other hand oligoanalgesia

- Because hydromorphone is more potent, at a much smaller milligram dose, physicians may be more likely to adequately treat pain by giving a dose of $1.5 \mathrm{mg}$ of hydromorphone vs. $10 \mathrm{mg}$ of morphine

- Hydromorphone causes little or no histamine release, and may be safely administered to patients who report a type 2 allergy to morphine (urticaria, pruritis, and facial flushing)

3. Should hydromorphone $1+1 \mathrm{mg}$ patient-driven protocol or other intravenous opioids at any dose (physician-driven protocol) be used for acute pain management?

We recommend a $1 \mathrm{mg}+1 \mathrm{mg}$ patient-driven protocol over other intravenous opioids in the emergency department (weak recommendation, low quality evidence)

This may be especially helpful for patients who are unable to clearly communicate their level of pain (acute mental status change, non-English speaking patients)

\section{Rationale [28, 31-33]}

- Hydromorphone has a quicker onset of action compared with morphine

- Hydromorphone is comparable in cost to morphine

- Morphine, with a longer onset of action and greater risk for dose stacking, places patients at a higher risk for toxicity (in the context of renal failure) and hypoventilation or, on the other hand, oligoanalgesia

\section{Oral analgesics}

\section{Should hydromorphone or oxycodone be used for acute pain?}

We are unable to comment on the superiority/inferiority of either of these drugs in treating acute pain

The only studies we identified that compared these drugs assessed the extended release forms of hydromorphone (Exalgo ${ }^{\circledR}$ ) and oxycodone Further research is needed to assess the immediate release forms of hydromorphone (e. g., Dilaudid ${ }^{\circledR}$ ) and oxycodone, and whether they have a role in treating acute pain in the emergency department

\section{Rationale [34, 35]}

- We only identified two studies comparing hydromorphone and oxycodone. They assessed the extended release forms. Both studies suggested no difference between the drugs in either pain relief or adverse effects

- We are unable to make recommendation about hydromorphone (PO) and oxycodone $(\mathrm{PO})$ in treating acute pain. Extended release forms appear to be equal in terms of pain relief and side effect profile (when dosed in an equal analgesic way 2:5) based on two RCTs (strong recommendation, very low quality evidence).

5. Should non-specific NSAIDs (e. g., ibuprofen) or codeineacetaminophen be used for mild-moderate acute pain?

We recommend non-COX specific NSAIDs over codeine-acetaminophen combinations

(weak recommendation, moderate quality evidence)

Rationale [36-38]

- $\quad$ The reported numbers needed to treat for naproxen and ibuprofen are 2.7 vs. 4.4 for codeine-acetaminophen

- NSAIDs have been shown to have a longer time to re-medication with a safer side effect profile. The number needed to treat for codeineacetaminophen was 6

- NSAIDs do not have the CNS depressing effects of codeine

- Certain genotypes may not metabolize or may hyper-metabolize codeine into morphine (due to a CYP2D6 polymorphism)

\section{Should COX-2 specific NSAIDs (e. g., celecoxib) or codeine-} acetaminophen be used for mild-moderate acute pain?

We recommend COX-specific NSAIDs over codeine acetaminophen combinations

(weak recommendation, moderate quality evidence)

\section{Rationale [36]}

- This is based on a Cochrane systematic review that compared NSAIDs and codeine-acetaminophen combinations with placebo in treating acute postoperative pain

- The number needed to treat for $400 \mathrm{mg}$ of celecoxib was 2.5 whereas that for $600 \mathrm{mg} / 60 \mathrm{mg}$ of acetaminophen/codeine was 3.9

- The average time to re-medication with celecoxib was $8.4 \mathrm{~h}$, whereas patients who used acetaminophen/codeine re-medicated in $4.1 \mathrm{~h}$

- The relative risk between these two drugs is 1

7. Should oxycodone-acetaminophen or codeine-acetaminophen be given to patients with acute pain in the ED?

We recommend oxycodone-acetaminophen as marginally superior to codeineacetaminophen

(weak recommendation, low quality evidence)

Rationale [39-41]

- This recommendation is based on two Cochrane reviews that compared each of these drugs with placebo

- There are few studies that directly compare these two drugs, especially in an adult emergency department setting

- However, the Cochrane reviews and single studies consistently show that oxycodone with acetaminophen is slightly better at relieving pain than acetaminophen-codeine 
- Because hydromorphone is more potent, at a much smaller milligram dose, physicians may be more likely to adequately treat pain by giving a dose of $1.5 \mathrm{mg}$ of hydromorphone vs. $10 \mathrm{mg}$ of morphine

- Hydromorphone causes little or no histamine release, and may be safely administered to patients who report a type 2 allergy to morphine (urticaria, pruritis, and facial flushing)

- This is superior to standard morphine and fentanyl dosing for a few reasons: Physicians tend to be concerned about giving patients more morphine than $5 \mathrm{mg}$ and often give small doses, e. g., $2.5 \mathrm{mg}$. A $1+1$ approach not only allows physicians to appropriately treat pain, but also requires fewer repeat orders

developed by the first author using the GRADE-Pro software. Subsequently the first author drafted recommendations for each of the seven PICO question using the data in the SoF tables. Once these drafts were complete, the second and third authors reviewed the SoF tables and reviewed the recommendations in an iterative process. These recommendations were then compiled into a succinct table.

\section{Results}

A total of 153 abstracts were screened for eligibility and 26 articles met eligibility criteria. Three articles compared fentanyl and morphine (PICO \#1); five articles assessed hydromorphone (PICO \#2), two of which analyzed the $1+1$ hydromorphone protocol (PICO \#3); three articles compared oral hydromorphone and oxycodone (PICO \#4); eight articles compared non-specific NSAIDs and codeine-acetaminophen (PICO \#5); two articles compared specific NSAIDs and codeine-acetaminophen (PICO \#6); and five articles compared oxycodone and codeine (PICO \#7).

The same reviewer assessed the full text articles and determined that 14 of the 26 offered quantitative results that could be analyzed using the GRADE-pro software [28-41]. The remaining 12 articles were excluded because they did not compare both drugs assessed in a PICO question [42-47], compared analgesics not addressed in the seven PICO questions (such as acetaminophen alone) $[48,49]$, used unconventional medication dosing [50, 51], used non-validated pain measurement scales [52, 53], and/or unusual study designs [50].

The 14 articles evaluated using the GRADE approach included eight randomized clinical trials, four systematic reviews, one retrospective cohort study, and one prospective clinical trial. An example of a SoF table developed for PICO number two is included (Table 3). Similar SoF tables were developed for each PICO question. Overall, the grading process supported the use of intravenous hydromorphone and fentanyl as superior to intravenous morphine for rapid and effective pain relief (weak recommendation, moderate quality evidence).
Oral NSAIDs, oxycodone, and hydromorphone are generally superior to codeine-acetaminophen combinations (weak recommendations, very-low quality evidence; due to bias, indirectness, and imprecision).

\section{Discussion}

Using the GRADE framework, we have synthesized and evaluated the medical literature surrounding analgesia delivery in the adult ED and developed clear and actionable recommendations for analgesic use based on graded recommendations. Despite using a thorough, systematic approach to reviewing the literature on each PICO question we identified only 14 articles that met our inclusion criteria. However, although the number of studies used to make our recommendations may seem low, our recommendations take into consideration the quality of evidence of the studies. Pragmatic derivations of the seven PICO questions, their recommendations, and the rationale for these recommendations are listed in Table 4 [28-41]. Furthermore, we also developed a flowchart as a suggested approach to analgesia in the ED (Fig. 1).

Despite the synthesis of information in this study there are also some limitations to consider. First, there is a limited body of literature analyzing the use and efficacy of analgesics in the ED setting. Even after searching multiple databases there were few studies conducted in the ED setting that compared one oral analgesic with another oral analgesic. As a result, many of our recommendations were limited by the fact that some studies were conducted in postoperative settings and subsequently downgraded due to indirectness. Second, there were difficulties making comparisons between different studies that compared the various oral agents. In particular, it was difficult to properly compare studies in which codeine-acetaminophen combinations were used as there is a significant amount of variability in the codeine to acetaminophen ratio in the various combinations of this oral analgesic. As a result, we were unable to clearly compare two studies if they differed in their codeine to acetaminophen ratios. Third, while the 


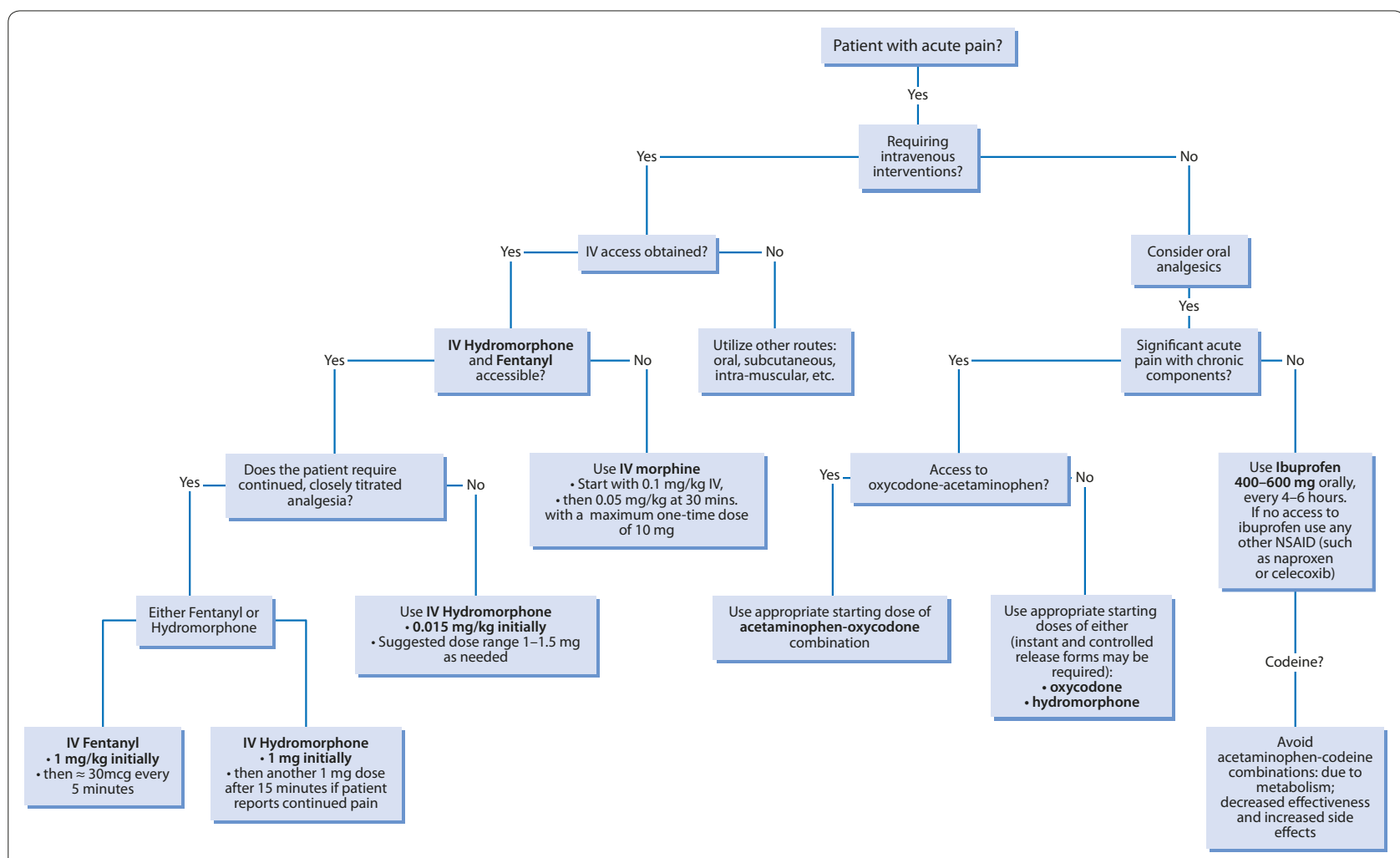

Figure 1. Suggested approach to acute pain in the emergency department. NSAID: non-steroidal anti-inflammatory drug; IV: intravenous.

authors collaborated extensively throughout this project, only one author was responsible for analyzing the article titles and abstracts. Finally, this review did not include the pediatric population in the literature search, and our recommendations do not extend to treating acute pain in children.

Despite these limitations this study also has a number of strengths. First, this study is extremely relevant to emergency physicians and health care workers who must treat acute pain, and to our knowledge it is the first GRADE-based evaluation of ED analgesia. Furthermore, this study serves as a model for clinician-researchers and administrators who want to promote evidence-based medicine in their clinical context. By conducting similar PICO question based analyses, successful knowledge translation from medical literature into clinical practice may be accomplished and, thereby, insure that treatments at the bedside remain current.

\section{Conclusion}

Despite the frequent occurrence of pain in ED patients, there are no ED-based syntheses and guidelines that compare analgesics commonly used in the ED. We have developed the first GRADE based recommendations for improving analgesia in the ED. Going forward, these findings can be used by ED clinicians and guideline panels to evaluate and develop analgesic order sets based on specific clinical presentations as well as drive the research agenda in ED analgesia.

These evidence-based guidelines have the potential to not only impact on patient morbidity, but also on health care costs and ED efficiency. As health care professionals with the role of treating emergent health problems, it is crucial that all emergency physicians have up-to-date, evidence-based knowledge to adequately treat acute pain.

\section{Competing interests}

The authors declare that they have no competing interests.

\section{List of abbreviations used}

ACCP: American College of Chest Physicians; Cl: Confidence interval; COX: cyclooxygenase; ED: emergency department; ERS: European Respiratory Society; GRADE: Grading Assessment, Development and Evaluation; ILCOR: International Liaison Committee on Resuscitation (ILCOR); IV: intravenous; MeSH: Medical Subject Headings; MMSE: mini mental state examination; NICE: National Institute for Clinical Excellence; NRS: numeric rating scale; NSAID: non-steroidal anti-inflammatory drug; PICO: Population-InterventionComparison-Outcome; RR: risk ratio; SCCM: Society of Critical Care Medicine; SoF: summary of findings; VAS: visual analogue; WHO: World Health

Organization.

\section{Acknowledgement}

We thank Dr Helen Lee-Robertson, librarian at the University of Calgary Health Sciences Center, for her contributions to this study.

Published: 19 March 2013 


\section{References}

1. Todd KH, Ducharme J, Choiniere M, Crandall CS, Fosnocht DE, Homel P, Tanabe P, PEMI Study Group: Pain in the emergency department: Results of the pain and emergency medicine initiative (PEMI) multicenter study. J Pain 2007, 8:460-466.

2. Silk J, Baird L, Ahern M, Holdgate A, Fry M: An emergency department's analysis of pain management patterns. Australian Emerg Nurs J 1999, 2:31-36.

3. Pines JM, Hollander JE: Emergency department crowding is associated with poor care for patients with severe pain. Ann Emerg Med 2008, 51:1-5.

4. Allione A, Melchio R, Martini G, Dutto L, Ricca M, Bernadi E, Pomero F, Menardo $V$, Tartaglino B: Factors influencing desired and received analgesia in emergency department. Intern Emerg Med 2011, 6:69-78.

5. Ducharme J: Proceedings from the first international symposium on pain research in emergency medicine: Foreword. Ann Emerg Med 1996, 27:399-403.

6. Cordell WH: Pain-control research opportunities and future directions. Ann Emerg Med 1996, 27:474-478.

7. Afilalo M, Tselios C: Pain relief versus patient satisfaction. Ann Emerg Med 1996, 27:436-438

8. Lee $\mathrm{G}$, Smith $\mathrm{S}$, Jennings $\mathrm{N}$ : Low acuity abdominal pain in the emergency department: Still a long wait. Int Emerg Nurs 2008, 16:94-100.

9. Finckh A, Robertson F, McDonell K, Welch S, Gawthorne J: Implementation of a guideline to improve prescription of analgesia for adult trauma patients in an emergency department. Australas Emerg Nurs J 2010, 13:25-29.

10. Wilson JE, Pendleton JM: Oligoanalgesia in the emergency department. Am J Emerg Med 1989, 7:620-623.

11. Singer A, Chisum E, Stark M: An educational intervention to reduce oligoanalgesia in the emergency department. Ann Emerg Med 2003, 42:S41-S41.

12. Decosterd I, Hugli O, Tamchès E, Blanc C, Mouhsine E, Givel JC, Yersin B, Buclin $\mathrm{T}$ : Oligoanalgesia in the emergency department: Short-term beneficial effects of an education program on acute pain. Ann Emerg Med 2007, 50:462-471

13. Calil AM, MattosPimenta CA, Birolini D: The "oligoanalgesia problem" in the emergency care. Clinics 2007, 62:591-598.

14. Motov SM, Khan AN: Problems and barriers of pain management in the emergency department: Are we ever going to get better? J Pain Res 2008 , 2:5-11.

15. Chen EH, Shofer FS, Dean AJ, Hollander JE, Baxt WG, Robey JL, Sease KL, Mills AM: Gender disparity in analgesic treatment of emergency departmen patients with acute abdominal pain. Acad Emerg Med 2008, 15:414-418

16. Rupp T, Delaney KA: Inadequate analgesia in emergency medicine. Ann Emerg Med 2004, 43:494-503.

17. Arendts $G$, Fry M: Factors associated with delay to opiate analgesia in emergency departments. J Pain 2006, 7:682-686.

18. Cordell WH, Keene KK, Giles BK, jones JB, Jones, JH, Brizendine EJ: The high prevalence of pain in emergency medical care. Am J Emerg Med 2002, 20:165-169.

19. Ducharme J: A prospective blinded study on emergency pain assessment and therapy. J Emerg Med 1995, 13:571-575.

20. Tcherny-Lessenot S, Karwowski-Soulié F, Lamarche-Vadel A, Ginsburg C, Brunet F, Vidal-Trecan G: Management and relief of pain in an emergency department from the adult patients' perspective. J Pain Symptom Manag 2003, 25:539-546.

21. Macintyre P, Scott DA, Schug SA, Visser EJ, Walker SM: Acute Pain Management: Scientific Evidence 2010

[http://www.anzca.edu.au/resources/college-publications/pdfs/Acute\%20 Pain\%20Management/books-and-publications/acutepain.pdf] Accessed October 2012

22. Morley PT, Atkins DL, Billi JE, Bossaert L, Callaway CW, de Caen AR, Deakin CD, Eigel B, Hazinski MF, Hickey RW, Jacobs I, Kleinman ME, Koster RW, Mancin ME, Montgomery WH, Morrison L, Nadkarni VM, Nolan JP, O'Connor RE, Perlman JM, Sayre MR, Semenko TI, Shuster M, Soar J, Wyllie J, Zideman D: Part 3: Evidence evaluation process: 2010 International Consensus on Cardiopulmonary Resuscitation and Emergency Cardiovascular Care Science With Treatment Recommendations. Circulation 2010 122:\$283-S8290.

23. Schunemann HJ, Cook D, Guyatt G: Methodology for antithrombotic and thrombolytic therapy guideline development: American College of Chest Physicians Evidence-based Clinical Practice Guidelines (8th edition). Chest
2008, 133(Suppl 6):110S-112S.

24. Guyatt GH, Oxman AD, Kunz R, Atkins D, Brozek J, Vist G, Alderson P, Glasziou $P$, Falck-Ytter Y, Schünemann HJ: GRADE guidelines: 2. Framing the question and deciding on important outcomes. J Clin Epidemio/ 2011, 64:395-400.

25. Guyatt G, Vist G, Falck-Ytter Y, Kunz R, Magrini N, Schunemann H: An emerging consensus on grading recommendations? Evid Based Med 2006, 11:2-4.

26. The GRADE Working Group: Organizations that have endorsed or that are using GRADE 2012. [http://www.gradeworkinggroup.org/society/index htm]. Accessed October 2012

27. Guyatt G, Oxman AD, Akl EA, Kunz R, Vist G, Brozek J, Norris S, Falck-Ytter Y DeBeer H, Jaeschke R, Rind D, Meerpohl J, Dahm P, Schünemann HJ: GRADE guidelines: 1. introduction-GRADE evidence profiles and summary of findings tables. J Clin Epidemio/ 2011, 64:383-394.

28. Patanwala AE, Keim SM, Erstad BL: Intravenous opioids for severe acute pain in the emergency department. Ann Pharmacother 2010, 44:1800-1809.

29. Galinski M, Dolveck F, Borron SW, Tual L, Van Laer V, Lardeur JY, Lapostolle F Adnet F: A randomized, double blind study comparing morphine with fentanyl in prehospital analgesia. Am J Emerg Med 2005, 23:114-119.

30. Fleischman RJ, Frazer DG, Daya M, Jui J, Newgard CJ: Effectiveness and safety of fentanyl compared with morphine for out-of-hospital analgesia. Prehosp Emerg Care 2010, 14:167-175.

31. Chang AK, Bijur PE, Gallagher EJ: Randomized clinical trial comparing the safety and efficacy of a hydromorphone titration protocol to usual care in the management of adult emergency department patients with acute severe pain. Ann Emerg Med 2011, 58:352-359.

32. Chang AK, Bijur PE, Campbell CM, Murphy MK, Gallagher EJ: Safety and efficacy of rapid titration using $1 \mathrm{mg}$ doses of intravenous hydromorphone in emergency department patients with acute severe pain: The"1 + 1" protocol. Ann Emerg Med 2009, 54:221-225

33. Chang AK, Bijur PE, Davitt M, Gallagher EJ: Randomized clinical trial comparing a patient-driven titration protocol of intravenous hydromorphone with traditional physician-driven management of emergency department patients with acute severe pain. Acute Pain 2009, 11:152-153.

34. Binsfeld H, Szczepanski L, Waechter S, Richarz U, Sabatowski R: A randomized study to demonstrate non-inferiority of once-daily OROS hydromorphone for moderate to severe chronic non-cancer pain. Pain Pract 2010, 10:404-415

35. Hale M, Tudor IC, Khanna S, Thipphawong J: Efficacy and tolerability of once-daily OROS hydromorphone and twice-daily extended-release oxycodone in patients with chronic, moderate to severe osteoarthritis pain: results of a 6-week, randomized, open-label, noninferiority analysis. Clin Ther 2007, 29:874-888.

36. Moore RA, Derry S, McQuay HJ, Wiffen PJ: Single dose oral analgesics for acute postoperative pain in adults. Cochrane Database Syst Rev 2011, CD008659.

37. Drendel AL, Gorelick MH, Weisman SJ, Lyon R, Brosseau DC, Kim MK: Randomized clinical trial of ibuprofen versus acetaminophen with codeine for acute pediatric arm fracture pain. Ann Emerg Med 2009, 54:553-560.

38. Innes GD, Croskerry P, Worthington J, Beveridge R, Jones D: Ketorolac versus acetaminophen-codeine in the ED treatment of acute low back pain. J Emerg Med 1998, 16:549-556.

39. Gaskell H, Derry S, Moore RA, McQuay HJ: Single dose oral oxycodone and oxycodone plus paracetamol for acute postoperative pain in adults. Cochrane Database Syst Rev 2009, CD002763

40. Toms I, Derry S, Moore RA, McQuay HJ: Single dose oral paracetamol with codeine for post-operative pain in adults. Cochrane Database Syst Rev 2009 CD001547.

41. Marco CA, Plewa MC, Buderer N, Black C, Roberts A: Comparison of oxycodone and hydrocodone for the treatment of acute pain associated with fractures: a double-blind randomised clinical trial. Acad Emerg Med 2005, 12:282-288.

42. Larijani GE, Goldberg MD, Fratz I, Warshal DP: Analgesic and hemodynamic effects of a single $7.5 \mathrm{mg}$ intravenous dose of morphine in patients with moderate-to-severe postoperative pain. Pharmacotherapy 2004 24:1675-1680

43. Kanowitz A, Dunn T, Kanowitz EM, Dunn WW, Vanbuskirk K: Safety and effectiveness of fentanyl administration for pre-hospital pain management. Prehosp Emerg Care 2006, 10:1-7. 
44. Chang AK, Bijur PE, Napolitano A, Lupow J, Gallagher EJ: Two milligrams intravenous hydromorphone is efficacious for treating pain but is associated with oxygen desaturation. J Opioid Manag 2009, 5:75-80.

45. Pergolizzi J, Böger RH, Budd K, Dahan A, Erdine S, Hans G, Kress HG, Langford R, Likar R, Raffa RB, Sacerdote P: Opioids and the management of chronic severe pain in the elderly: consensus statement of an International Expert Panel with focus on the six clinically most often used World Health Organization Step III opioids (buprenorphine, fentanyl, hydromorphone, methadone, morphine, oxycodone). Pain Pract 2008 8:287-313.

46. Clark E, Plint AC, Correll R, Gaboury I, Passi B: A randomized, controlled trial of acetaminophen, ibuprofen, and codeine for acute pain relief in children with musculoskeletal trauma. Pediatrics 2007, 119:460-467.

47. Derry S, Barden J, McQuay HJ, Moore RA: Single dose oral celecoxib for acute postoperative pain in adults. Cochrane Database Syst Rev 2008, CD004233.

48. McNicol ED, Strassels S, Goudas L: NSAIDS or paracetamol, alone or combined with opioids for cancer pain. Cochrane Database Syst Rev 2011, CD005180.

49. Holdgate A, Pollock T: Nonsteroidal anti-inflammatory drugs (NSAIDS) versus opioids for acute renal colic. Cochrane Database Syst Rev 2004, CD004137.

50. Miner JR: Randomized double-blind placebo controlled crossover study of acetaminophen, ibuprofen, acetaminophen/hydrocodone, and placebo for the relief of pain from a standard painful stimulus. Acad Emerg Med 2009, 16:911-911.

51. White PF, Joshi GP, Carpenter RL, Fragen RJ: A comparison of oral ketorolac and hydrocodone-acetaminophen for analgesia after ambulatory surgery. Anesth Analg 1997, 85:37-43.

52. Gimbel JS, Brugger A, Zhao W, Verburg KM, Geis GS: Efficacy and tolerability of celecoxib versus hydrocodone/acetaminophen in the treatment of pain after ambulatory orthopedic surgery in adults. Clin Ther 2001, 23:228-241.

53. Litkowski L, Christensen SE, Adamson DN, Van Dyke T, Han SH, Newman KB: Analgesic efficacy and tolerability of oxycodone $5 \mathrm{mg} / \mathrm{ibuprofen} 400 \mathrm{mg}$ compared with those of oxycodone $5 \mathrm{mg} /$ acetaminophen $325 \mathrm{mg}$ and hydrocodone $7.5 \mathrm{mg} /$ acetaminophen $500 \mathrm{mg}$ in patients with moderate to severe postoperative pain: A randomized, double-blind, placebocontrolled, single-dose, parallel-group study in a dental pain model. Clin Ther 2005, 27:418-429.

doi:10.1186/cc12521

Cite this article as: Lipp C, et al:: Analgesia in the emergency department: a GRADE-based evaluation of research evidence and recommendations for practice. Critical Care 2013, 17:212. 